\title{
Compressed sensing for imaging transcriptomics
}

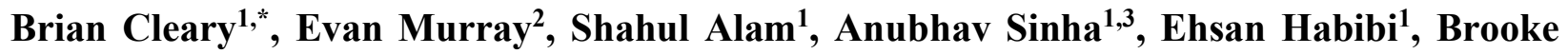 \\ Simonton ${ }^{1}$, Jon Bezney ${ }^{1}$, Jamie Marshall², Eric S. Lander ${ }^{2,4,5, *}$, Fei Chen ${ }^{1,2, *}$, Aviv Regev 1,4,6,*
}

\begin{abstract}
Affiliations
${ }^{1}$ Klarman Cell Observatory, Broad Institute of MIT and Harvard, Cambridge, MA 02142, USA

${ }^{2}$ Broad Institute of MIT and Harvard, Cambridge, MA 02142, USA

${ }^{3}$ Harvard-MIT Division of Health Sciences and Technology, Cambridge, MA 02139 USA

${ }^{4}$ Department of Biology, Massachusetts Institute of Technology, Cambridge, MA 02139, USA

${ }^{5}$ Department of Systems Biology Harvard Medical School, Boston, MA 02125, USA

${ }^{6}$ Howard Hughes Medical Institute, Chevy Chase, MD, USA

*Correspondence should be addressed to bcleary@broadinstitute.org, chenf@broadinstitute.org, aregev@,broadinstitute.org, and lander@broadinstitute.org
\end{abstract}




\begin{abstract}
Gaining a systematic molecular understanding of tissue physiology in health and disease will require the ability to rapidly profile the abundances of many genes at high resolution over large tissue volumes. Many current methods of imaging transcriptomics are based on single-molecule fluorescent in situ hybridization, with barcodes to allow multiplexing across genes. These approaches have serious limitations with respect to (i) the number of genes that can be studied and (ii) imaging time, due to the need for high-resolution to resolve individual signals. Here, we show that both challenges can be overcome by introducing an approach that leverages the biological fact that gene expression is often structured across both cells and tissue organization. We develop Composite In Situ Imaging (CISI), that combines this biological insight with algorithmic advances in compressed sensing to achieve greater efficiency. We demonstrate that CISI accurately recovers the spatial abundance of each of 37 individual genes in the mouse primary motor cortex (MOp) from 10 composite measurements and without the need for spot-level resolution. CISI achieves the current scale of multiplexing with an order of magnitude greater efficiency, and can be leveraged in combination with existing methods to multiplex far beyond current scales.
\end{abstract}


Tissue and organ function rely on the organization of cells and molecules in specific spatial structures. In order to understand these structures and how they relate to tissue function in health and disease, we would ideally be able to rapidly profile gene expression over large tissue volumes. To this end, in recent years multiple molecular assays have been developed that can image from a dozen to 100 individual proteins (Angelo et al., 2014; Goltsev et al., 2018; Keren et al., 2018) or RNAs (Chen et al., 2015; Choi et al., 2018; Codeluppi et al., 2018; Shah et al., 2016, 2017; Wang et al., 2018a, 2018b) in a sample at single-cell resolution.

The most commonly used, highly multiplexed methods for imaging RNA are based on singlemolecule fluorescence in situ hybridization (smFISH) (Raj et al., 2008), with rounds of staining and stripping, and a variety of barcoding strategies to increase multiplexing (Chen et al., 2015; Choi et al., 2018; Codeluppi et al., 2018; Shah et al., 2016, 2017; Wang et al., 2018a, 2018b). In "linear" barcoding strategies (e.g., osmFISH (Codeluppi et al., 2018) or in situ HCR (Choi et al., 2018)), each color in each round corresponds to one gene. The number of genes $G$ that can be measured with $c$ colors and $r$ rounds is thus $G=c r$. In "combinatorial" strategies, such as MERFISH (Chen et al., 2015; Wang et al., 2018a) or Seq-FISH (Shah et al., 2016, 2017), gene identity is encoded by a sequence of colors over multiple rounds - that is, many genes may share a given color in a given round, but each gene is encoded by a unique sequence of colors across the rounds. The number of genes $G$ that can be measured with $c$ colors and $r$ rounds is thus $G=c^{r}-$ ignoring additional rounds needed for error correction. (The number of colors available is typically 3 or 4.$)$ 
Multiplex methods have provided an unprecedented tool for tissue biology and histopathology, but they typically measure fewer than $1 \%$ of genes, necessitate choosing a gene-expression signature, and can require a week or more to collect these data in a single tissue section. Ideally, it would be possible to quickly generate data on thousands of gene abundance levels in large tissue volumes, perhaps even entire organs.

Notably, existing barcoding methods ignore prior knowledge or biological principles: each spot is decoded independently, without using any 'local' information (such as gene-expression information at nearby spots). This choice leads to two fundamental limitations on scalability. First, both linear and combinatorial quantification requires imaging at high magnification (up to 100x) so that individual RNA molecules appear as bright, well-separated spots, so that their individual identities can be decoded from the hybridization images. High-resolution image acquisition over large volumes is a major time bottleneck. Second, there are limitations on the number of genes. In linear barcoding, it is not feasible to substantially increase the number $G$ of genes assayed, because the number of rounds of imaging scales with $G$ (100-fold more genes requires 100 -fold more rounds) and with combinatorial barcoding, increases are limited by optical crowding (spatial overlap between fluorescent spots), because the number of spots scales with $G$. Recent efforts to ameliorate this latter issue with sparser combinatorial barcodes increase the number of rounds of hybridization and, so far, result in a relatively high rate of false positives (Eng et al., 2019).

We reasoned that a biology-informed strategy could be more efficient, by incorporating knowledge about the principles of gene expression patterns. Because many genes are co-regulated, measurements of one gene give information about the likely abundances of others. In such cases, 
one might infer the expression of many individual genes from a much smaller number of composite measurements of gene abundance - mathematically defined as linear combinations of gene abundance levels - consisting of combined signal from multiple genes on the same channel. That is, instead of measuring the level of multiple genes but each of them separately (i.e., in one channel), we should use each channel to measure the composite (sum) abundances of multiple genes in each channel, and later be able to decompress and determine individual gene levels by leveraging the biological insights that genes are co-regulated in modules. We have previously published the theoretical foundations of this strategy, based on the mathematics of compressed sensing (Cleary et al., 2017), which describes how under-sampled composite data can be decompressed to recover structured, high-dimensional expression signals for individual genes.

Here, we develop such a scheme, Composite In Situ Imaging (CISI), implement it in a lab method and computational algorithm, and show that it improves the current throughput of convenient linear barcoding methods by at least an order of magnitude. Our implementation of CISI consists of four steps (Fig. 1a).

(1) Create a dictionary of gene-expression modules. To study the spatial expression pattern of $G$ genes in tissue samples, we first obtain single-cell profiles (e.g., from single cell RNA-Seq (scRNA-Seq)) from comparable samples to identify co-expression patterns among the selected genes and to compute a dictionary consisting of $M$ sparse gene-expression modules (i.e., each module is a sparse vector of nonnegative coefficients for the genes) such that the single-cell profiles can be well approximated by $D$-sparse linear combinations of the modules (i.e., involving 
at most D non-zero weights) (Cleary et al., 2017). Below, we use $D=3$ and explain how it was chosen empirically.

(2) Select composite measurements. We next select $K$ composite measurements that enable accurate recovery of the chosen genes. Each composite measurement consists of probes for a subset of genes, corresponding to a linear combination of gene abundance. To select the compositions and numbers of measurements needed for accurate recovery, we simulate compressed sensing in the single-cell data: we first generate a random assignment of genes to measurements, simulate composite measurements as the sum of those genes' abundances, and compute the recovered (decompressed) profiles. We use simulations to test parameters for (1) the total number of measurements $K$, (2) the maximum number of measurements in which each gene was included, (3) the individual genes for each measurement, and (4) the size $M$ of the module dictionary and sparsity $D$ of the linear combinations. To simplify laboratory implementation, we considered only measurement compositions consisting of binary weights, where each gene was either not included, or included in equal proportion. For each combination of design parameters (1) and (2), we generate many simulated compositions and compute the recovered profiles from each, and then select compositions that most accurately recover the original expression levels (Methods).

(3) Generate image data. We then synthesize probes for each gene, and create composite probes for each composite measurement by mixing the probes according to the coefficients in the composite design. (Since the weights are binary, the probes for each gene included in a composition are mixed in equal proportions.) We hybridize the composite probes using the linear 
barcoding approach: in each round, we label $c$ composite probes with distinct colors. (For validation, we can include one or more additional cycles to directly measure a subset of individual genes.)

(4) Computational inference of gene-expression in each cell. Finally, we infer the gene expression patterns in the image, using one of two approaches. In the first, the image is segmented into cells. In each segmented cell, we add up the intensity of each color in each round to get a vector $\mathrm{y}$, corresponding to the composite measurements. We then solve a sparse optimization problem to estimate the gene module activities, $\mathrm{w}$, and individual gene abundances, $\mathrm{x}=\mathrm{Uw}$, given the composite designs, $A$, and a gene module dictionary, $U$. (That is, we solve for w in $\mathrm{y}=\mathrm{AUw}$ and then calculate $\mathrm{x}$; this is the core optimization problem of compressed sensing).

In an alternative approach, we analyze the image without cell segmentation or explicit spot detection by using a convolutional autoencoder to infer individual gene abundances at each pixel in the image. Specifically, we use a convolutional autoencoder to compute a low-dimensional, encoded representation of each image, and perform decompression in the encoded latent space (Fig. 1b, Methods). In the segmentation-free algorithm we developed for this purpose, we first train a convolutional encoder to represent each of the composite images in a lower-dimensional space. This effectively aggregates local pixel intensities according to data-driven features. At the same time, we train a decoder that can take these encoded representations as input, and then output images that match the originals. Next, for decompression, we take the $K$-channel encoded representation of each tissue section as input (each channel corresponding to one of the $K$ composite images). For each node in the encoded representation of a given tissue section, we then 
solve a sparse optimization problem to estimate gene module activities, and compute the encoded representation of the (unobserved) image for each individual gene (i.e., we decompress the encoded representation from $K$ to $G$ channels). We then decode the encoded representation of each unobserved gene, outputting $G$ individual images. During this optimization we include in the loss function the error between the re-composed individual genes and the original composites at both the encoded and decoded layers (among other constraints and regularizations; Methods).

CISI offers two important advantages. Like combinatorial barcoding, CISI requires exponentially fewer rounds $r$ of hybridization than linear barcoding $\left(\mathrm{r}_{\mathrm{CISI}}=\mathrm{O}(\mathrm{D} \ln (\mathrm{M}) / \mathrm{c}), \mathrm{r}_{\text {combinatorial }}=\right.$ $\ln (\mathrm{G}) / \ln (\mathrm{c})$, and $\mathrm{r}_{\text {linear }}=\mathrm{G} / \mathrm{c}$; we estimate that, in practice, that the number of rounds with either method will be comparable, with $\mathrm{r}_{\mathrm{CISI}} / \mathrm{r}_{\text {combinatorial }}$ typically between $1 / 3$ and 3 ; Methods). But unlike combinatorial barcoding, CISI does not require spot-level resolution, and thus allows for faster imaging over large areas: whereas individual spots are often imaged between 60-100x magnification, CISI can be imaged from 10-40x, allowing for imaging that is $2.25-100$ fold faster in two dimensional scanning.

To demonstrate CISI in practice, we applied it in the mouse primary motor cortex (MOp). We analyzed a set of 31,516 previously published single-nucleus RNA-Seq (snRNA-Seq) profiles from MOp (https://biccn.org/data). We chose to study $G=37$ genes, consisting of 30 genes that are markers of either broader (excitatory and inhibitory neurons, and various glial cells) or narrower (e.g., layer specific inhibitory neurons) subtypes (Supplementary Table 1, Supplementary Fig. 1), and 7 additional genes that were co-expressed with these markers. In the 27,491 cells in which at least 1 of the 37 genes was detected, the effective number of genes 
expressed (out of 37, using Shannon Diversity) per cell was 2.87. Given dropouts in snRNA-seq, this is likely an underestimate of true expression.

We then learned a sparse modular representation of the expression of the 37 genes in the 27,491 cells. For cells with only 1 or 2 of the genes expressed, we could trivially represent their expression with 1 or 2 parameters (corresponding to singleton gene modules). For cells with more genes expressed, it is more efficient to represent expression in terms of modules of co-expressed genes. We used our previously published method, Sparse Module Activity Factorization (SMAF (Cleary et al., 2017)), to identify a dictionary of $M=80$ modules. The modules effectively consist of 1 to 6 genes (2.66 on average; Supplementary Table 2), such that the expression of each of the 37 genes in each of 27,491 cells can be represented with a linear combination of 3 or fewer modules with $94.3 \%$ correlation. This representation was good ( $88 \%$ correlation), even in cells with greater than 5 of 37 genes expressed (Supplementary Fig. 2a). On average, each cell was described by the activity of 1.72 modules, and most cells were very accurately described (correlation $>95 \%$ ) by just 1 or 2 modules (Supplementary Fig. 2b).

We then used the simulation procedure described above to develop barcodes and composite measurements that would allow us to learn the modules in each cell (or small region of a tissue section), and subsequently approximate the 37 genes. As expected, performance improved with increasing numbers of measurements (criterion (1)), leveling off around 10 measurements (Supplementary Fig. 3a). The best compositions using each gene in a maximum of 2, 3, or 4 measurements (criterion (2)) resulted in recovered profiles that were $78 \%, 84 \%$, and $87 \%$ correlated with the original profiles (Supplementary Fig. 3b). We also considered scalability of 
probe synthesis. In particular, the number of gene-composition combinations that we would need to synthesize when each gene was included in 2, 3, or 4 measurements was 74,97 , and 120, respectively. Balancing the performance of the different designs and the estimated costs, we selected the best performing set of 10 composite measurements, with each gene included in up to 3 compositions (Supplementary Table 3). Using these parameters, we found that simulation refined performance from a median correlation of $76 \%$ to $84 \%$ with the best performing selection, with these compositions including between 6 and 13 genes (Supplementary Table 3).

We synthesized, pooled, and successfully tested the 10 selected compositions. We designed probe pairs targeting multiple regions of each gene for fluorescent in situ hybridization with HCR amplification (HCR-FISH (Choi et al., 2018), Methods, Supplementary Table 4), where each oligonucleotide contains a gene targeting sequence and a barcode that determines the channel (color) of the HCR amplified signal. We assigned each of the 10 compositions to one of three colors, to be imaged during $31 / 3$ rounds, pooling the assignment barcoded probes into the 10 compositions. For each of the 10 compositions, we tested these pools by imaging each gene individually, along with the pool of probes for the composition. We simulated composite images by merging into a composition the images acquired individually for each gene. The real and simulated composite images agreed well visually (Fig. 2a), and had 90.1\% correlation between integrated intensity values in segmented cells (on average, across the 10 compositions).

Next, we generated a large imaging dataset, using our validated composite probe libraries, together with probes for individual genes measured and used only for later confirmation. In each tissue section comprising $\sim 2,500-3,000$ cells, we first imaged the 10 composite measurements over $31 / 3$ 
rounds. Using the remaining two colors in the fourth round, and all three colors in a fifth round of imaging, we also directly measured each of up to five individual genes, for subsequent validation purposes. We repeated this in 8 tissue sections, picking different individual genes each time, such that in total we directly measured each of the 37 genes individually along with the compressed measurements (Supplementary Table 5).

We decompressed the experimental data with our segmentation-based and segmentation-free algorithms, and evaluated the accuracy of our results in several ways. First, the decompressed images for several genes corresponded well to their known distinct and readily identifiable spatial expression patterns, available as reference images from the Allen Brain Atlas (Fig. 2c). For example, these included: Slc17a7, which is broadly expressed in excitatory neurons throughout MOp; Vip, a marker of a subtype of inhibitory neurons, which is expressed more frequently in layer 2/3; and Sst, a marker of another inhibitory neuron subtype, which is expressed more frequently in layer 5 .

Second, the decompressed images agreed well with the direct measurements of each gene made in the same section, for genes expressed in both rare and common cell types, and in cells of varying morphologies (Fig. 2c and Supplementary Fig. 4). The correlation between direct and recovered (decompressed) measurements based on integrated signal intensity in segmented cells was high, either when using recovered values from the segmentation-free autoencoding algorithm (83.6\%) or when using decompression from segmented cells $(88.4 \%)$. (We expected the autoencoding algorithm to perform slightly worse by this metric, since it is not optimized for the segmentation 
masks.) Both are in line with the simulations used to design our measurements, which predicted a correlation of $84 \%$.

Notably, the segmentation-free autoencoder out-performs the segmentation-based algorithm for genes whose expression does not necessarily follow simple patterns (Supplementary Fig. 5). The segmentation-based approach omits regions of the image outside of successfully segmented cells, and can result in loss of morphological information, since the output typically consists of filled polygons with uniform intensity. Conversely, the autoencoding algorithm does not omit any regions, and retains morphology by data-driven convolutional features. As a result, while genes like Vtn have expression patterns easily captured by the filled polygons of segmented cells, others such as Flt1 and Parm1 are well-described by autoencoding, but not by segmentation (Fig. 2d,

\section{Supplementary Fig. 5).}

We analyzed cell-type composition of the autoencoding results, by segmenting cells post hoc (on decompressed images) and clustering the segmented cells based on the integrated intensity values across genes (Methods). Based on the markers in each cluster, neurons comprised about half of all (successfully segmented) cells: $33.3 \%$ of cells in 9 excitatory clusters, and $16.9 \%$ of cells in 6 inhibitory clusters. In addition, we find 4 clusters of oligodendrocytes and oligodendrocyte precursor cells (16.8\%), 3 clusters of astrocytes (12.8\%), 3 clusters of microglia $(9.1 \%), 2$ clusters of smooth muscle cells (6.5\%) and 2 clusters of endothelial cells (4.4\%). These in situ results are comparable with the representation of these cell subsets in snRNA-seq, albeit somewhat enriched in glial and depleted in endothelial cells: 44.5\% excitatory, $14.5 \%$ inhibitory, $8.9 \%$ 
oligodendrocyte / OPC, 10.4\% astrocyte, $4.6 \%$ microglia, $1.9 \%$ smooth muscle cells, and 15\% endothelial.

The points of inaccurate recovery were relatively predictable and consistent between the two algorithms, with some false positives for several genes, but few false negatives (Supplementary Fig. 6a). Eight genes had some false positive expression patterns in the recovered images that were absent from the direct measurements (Supplementary Table 6). In each case, the false positive signals co-occurred with a gene that had probes included in overlapping measurements. For instance, false positives for Hmhal are found in cells that express Slc17a7 (Supplementary Fig. 6c, left). Hmha1 is a member of two compositions, both of which also included Slc17a7, which is additionally included in a third composition.

We developed a simple heuristic to address this, by reducing false positives at the expense of some false negatives. For the 104 pairs of co-measured genes (i.e., that co-occur in more than one composition) that were not correlated $(<10 \%)$ in snRNA-Seq, we set the expression of one of the two genes to zero whenever they were co-expressed in recovered images (Methods). To select which gene to adjust, we calculate the correlation between the 10 composite measurements in a cell, and the pattern of measurements for each of the two genes (e.g., the binary vector indicating which measurements included the gene), and then adjust to zero the gene with the lower correlation. Applying this simple rule reduced false positives and improved the overall correlation from $83.6 \%$ to $88.6 \%$ with autoencoding, and from $88.4 \%$ to $91.6 \%$ with segmentation (Supplementary Fig. 6a,b). 
Finally, using these adjusted values, we found that decompressed measurements are substantially less sparse than snRNA-Seq, while preserving the co-expression programs observed in snRNASeq. As previously observed with osm-FISH (Codeluppi et al., 2018), the degree of sparsity is much greater in snRNA-Seq than in our decompressed measurements, with 2.87 genes detected on average in each cell in snRNA-Seq vs. 5.96 based on decompressed images (6.49 with segmentation) (Supplementary Fig. 7a). To compare co-expression patterns, we clustered cells in the (post hoc segmented) decompressed data (as discussed above) and in snRNA-Seq (using only the 37 genes), finding 29 and 28 clusters, respectively. Most clusters had expression signatures that were highly correlated with a counterpart in snRNA-Seq, and had identical sets of marker genes (i.e., the gene with the highest normalized expression in each cluster) (Supplementary Fig. 7b).

In conclusion, CISI addresses the two key bottlenecks in imaging transcriptomics: increasing the number of genes studied per round of hybridization and decreasing the time needed to scan large tissue volumes per round. In the results here, we improved the multiplexing efficiency by 3.7-fold (by assaying 37 individual genes with 10 composite measurements) and reduced the imaging time by 6.25 -fold (by using 40x vs. 100x magnification) compared to state-of-the-art methods that achieve a similar scale of multiplexing with osmFISH (Codeluppi et al., 2018). In principle, CISI could also be used to increase multiplexing with combinatorial labeling (with each combinatorial barcode corresponding to one composite), although high-magnification imaging would be needed to resolve (and decode) each individual spot, and fluorescence crowding would still pose a challenge. 
The results here point towards the possibility of greatly increased throughput in imaging transcriptomics. More broadly, CISI is in a class of methods that leverage algorithmic insights and biological structure to be more efficient in generating and interpreting data. Further applications in this class could increase multiplexed protein detection with antibodies, make single cell and single nucleus RNA-seq more efficient by sequencing small pools of cells, or efficiently study genetic perturbations by leveraging common outcomes across experiments. 
Acknowledgements: We thank A. Hupalowska and L. Gaffney for help with figures, S. Farhi, Y. Eldar and members of the Fei, Regev, and Lander labs for helpful discussion, and BICCN for open sharing of data pre-publication. Work was supported by the Klarman Cell Observatory, HHMI, NHGRI Center of Excellence in Genome Science (CEGS; RM1HG006193), and BRAIN Initiative U19 grant (1U19MH114821) (AR) and the Eric and Wendy Schmidt Fellows Program at the Broad Institute (FC). Author Contributions: $\mathrm{BC}, \mathrm{FC}$, and $\mathrm{AR}$ conceived the study. $\mathrm{EM}$ and $\mathrm{AS}$ performed experiments with assistance and feedback from BS, JB, and JM. BC, SA, and EH performed snRNA-Seq data analysis, and developed the image processing pipeline. BC developed and implemented the decompression algorithms. BC, AR, FC, ESL, and BS wrote the manuscript with input from all authors. Declaration of interests: A.R. is a founder of Celsius Therapeutics and a member of the SAB of Syros Pharmaceuticals, Driver Group and ThermoFisher Scientific. E.S.L. serves on the Board of Directors for Codiak BioSciences and Neon Therapeutics, and serves on the Scientific Advisory Board of F-Prime Capital Partners and Third Rock Ventures; he also serves on the Board of Directors of the Innocence Project, Count Me In, and Biden Cancer Initiative, and the Board of Trustees for the Parker Institute for Cancer Immunotherapy. 


\section{References:}

Abràmoff, M.D., Magalhães, P.J., and Ram, S.J. (2004). Image processing with imageJ.

Biophotonics Int.

Angelo, M., Bendall, S.C., Finck, R., Hale, M.B., Hitzman, C., Borowsky, A.D., Levenson,

R.M., Lowe, J.B., Liu, S.D., Zhao, S., et al. (2014). Multiplexed ion beam imaging of human

breast tumors. Nat. Med. 20, 436-442.

Chen, K.H., Boettiger, A.N., Moffitt, J.R., Wang, S., and Zhuang, X. (2015). Spatially resolved, highly multiplexed RNA profiling in single cells. Science (80-. ). 348, aaa6090-aaa6090.

Choi, H.M.T., Schwarzkopf, M., Fornace, M.E., Acharya, A., Artavanis, G., Stegmaier, J.,

Cunha, A., and Pierce, N.A. (2018). Third-generation in situ hybridization chain reaction:

multiplexed, quantitative, sensitive, versatile, robust . Development 145, dev165753.

Cleary, B., Cong, L., Cheung, A., Lander, E.S., and Regev, A. (2017). Efficient Generation of

Transcriptomic Profiles by Random Composite Measurements. Cell 171, 1424-1436.e18.

Codeluppi, S., La Manno, G., van Lunteren, J.A., Linnarsson, S., Zeisel, A., Borm, L.E.,

Svensson, C.I., Zeisel, A., La Manno, G., van Lunteren, J.A., et al. (2018). Spatial organization

of the somatosensory cortex revealed by osmFISH. Nat. Methods 15, 932-935.

Eng, C.H.L., Lawson, M., Zhu, Q., Dries, R., Koulena, N., Takei, Y., Yun, J., Cronin, C., Karp,

C., Yuan, G.C., et al. (2019). Transcriptome-scale super-resolved imaging in tissues by RNA

seqFISH+. Nature.

Goltsev, Y., Samusik, N., Kennedy-Darling, J., Bhate, S., Hale, M., Vazquez, G., Black, S., and

Nolan, G.P. (2018). Deep Profiling of Mouse Splenic Architecture with CODEX Multiplexed

Imaging. Cell 203166.

Keren, L., Bosse, M., Marquez, D., Angoshtari, R., Jain, S., Varma, S., Yang, S.-R., Kurian, A., 
Van Valen, D., West, R., et al. (2018). A Structured Tumor-Immune Microenvironment in Triple Negative Breast Cancer Revealed by Multiplexed Ion Beam Imaging. Cell 174, 1373-1387.e19. McQuin, C., Goodman, A., Chernyshev, V., Kamentsky, L., Cimini, B.A., Karhohs, K.W., Doan, M., Ding, L., Rafelski, S.M., Thirstrup, D., et al. (2018). CellProfiler 3.0: Next-generation image processing for biology. PLoS Biol.

Raj, A., van den Bogaard, P., Rifkin, S.A., van Oudenaarden, A., and Tyagi, S. (2008). Imaging individual mRNA molecules using multiple singly labeled probes. Nat. Methods.

Shah, S., Lubeck, E., Zhou, W., and Cai, L. (2016). In Situ Transcription Profiling of Single Cells Reveals Spatial Organization of Cells in the Mouse Hippocampus. Neuron.

Shah, S., Lubeck, E., Zhou, W., and Cai, L. (2017). seqFISH Accurately Detects Transcripts in Single Cells and Reveals Robust Spatial Organization in the Hippocampus. Neuron.

Wang, G., Moffitt, J.R., and Zhuang, X. (2018a). Multiplexed imaging of high-density libraries of RNAs with MERFISH and expansion microscopy. Sci. Rep.

Wang, X., Allen, W.E., Wright, M.A., Sylwestrak, E.L., Samusik, N., Vesuna, S., Evans, K., Liu, C., Ramakrishnan, C., Liu, J., et al. (2018b). Three-dimensional intact-tissue sequencing of single-cell transcriptional states. Science (80-. ). 


\section{Figures}

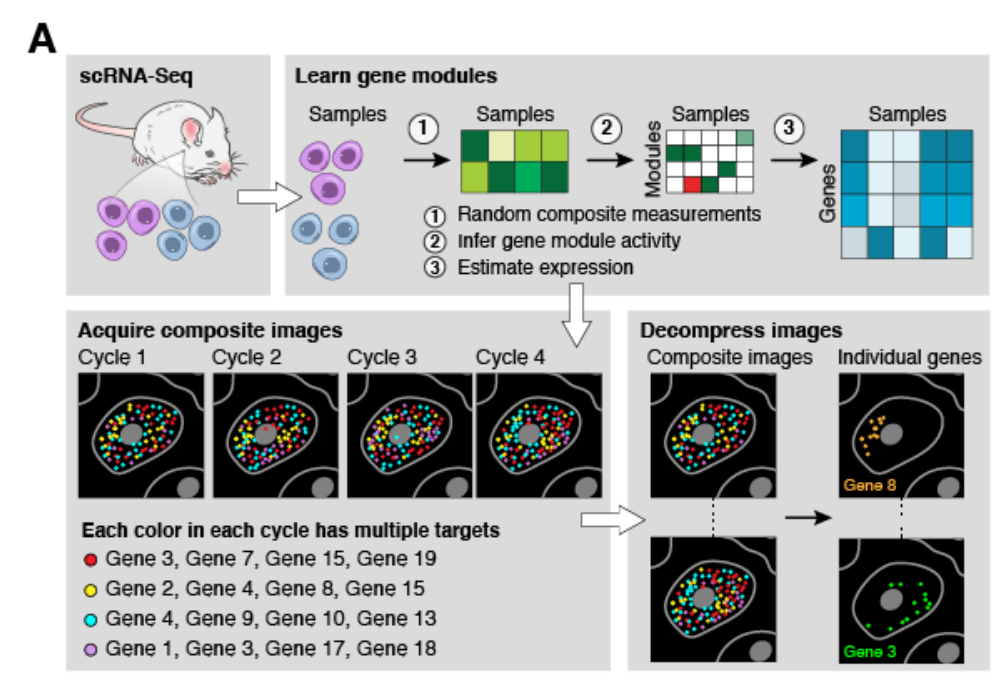

\section{Figure 1}

B
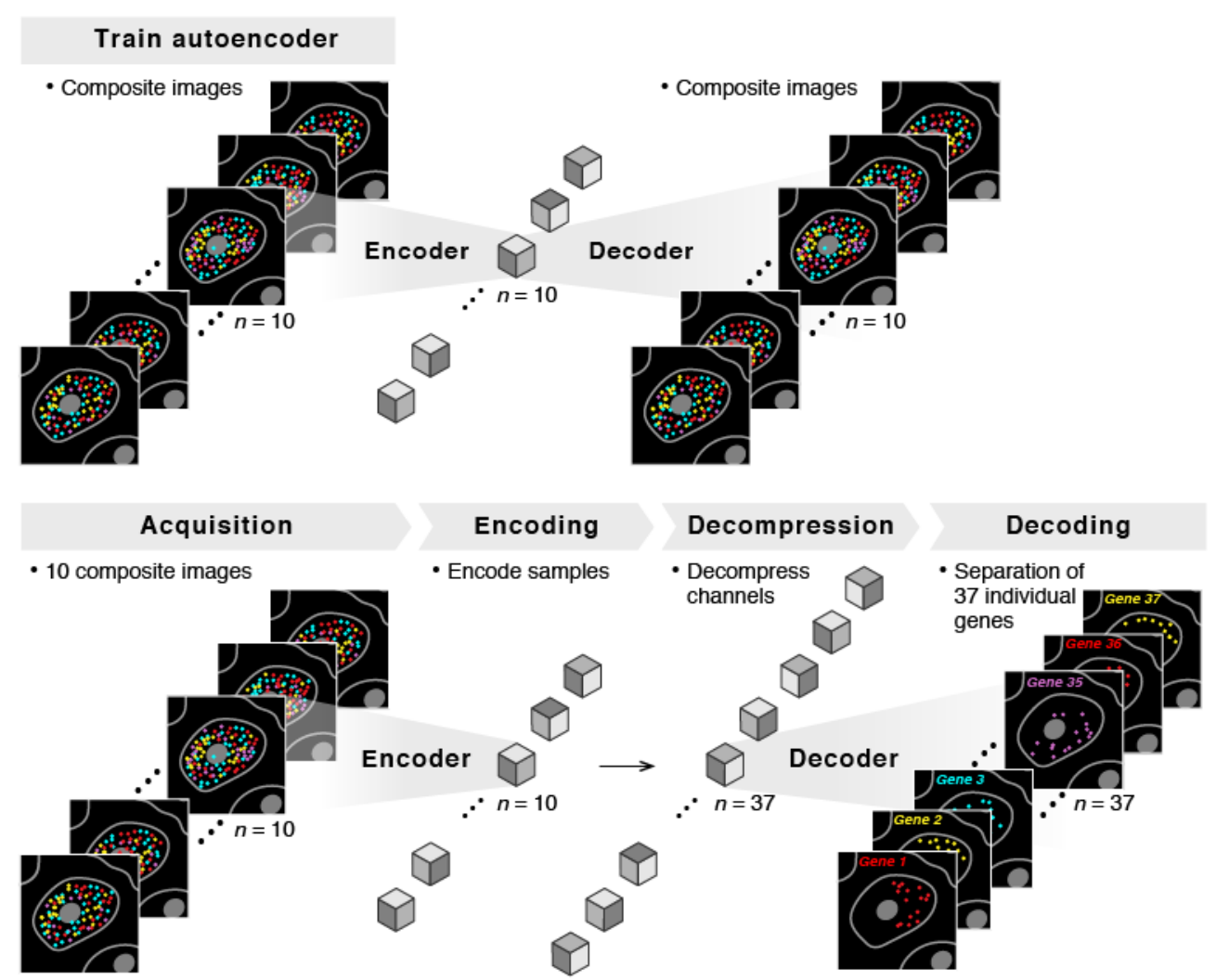

Figure 1. Composite In Situ Imaging (CISI)

(a) Method overview. snRNA-Seq data (top left) is first analyzed (top right) to learn a dictionary

of gene modules, simulate compressed sensing, and select measurement compositions to be used 
in CISI experiments (bottom left). In a CISI experiment, in each color in each round of imaging, probes for every gene in a given composite measurement are hybridized simultaneously. The process is repeated for different compositions over several cycles of stripping and hybridization (bottom left). Finally, composite images are then decompressed computationally (bottom right) to recover individual images for each gene. (b) Segmentation-free decompression. Top: An autoencoder is first trained on the composite images, with each composite measurement corresponding to one channel. Bottom: Once the autoencoder is trained, the composite images are encoded ("Encoding"), then decompressed to approximate the encoded representation for the unobserved image of each individual gene ("Decompression”), and the pre-trained decoder is used to recover individual images for each gene ("Decoding"). 


\section{Figure 2}

A
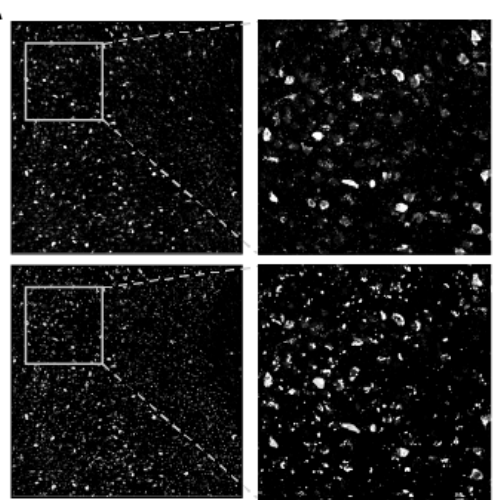

Composition 2
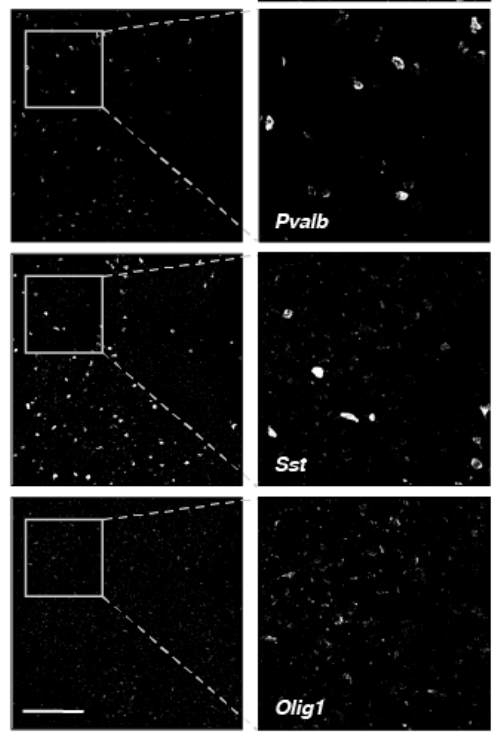

that comprise
B

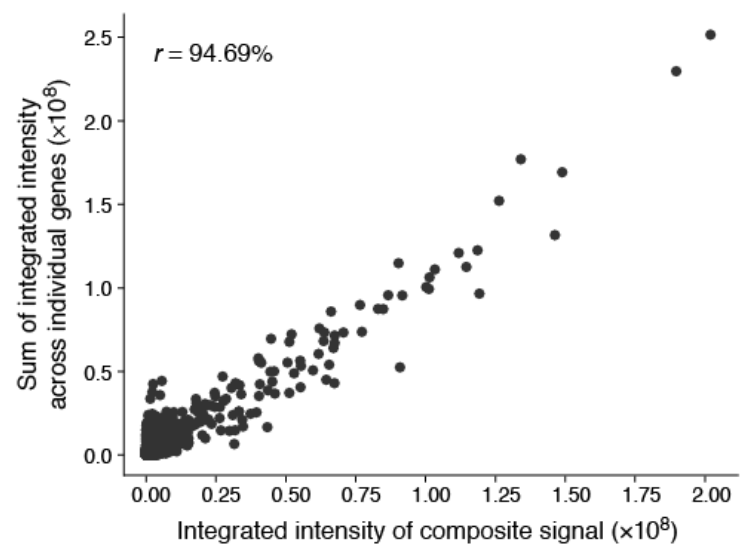

Three samples of

individual genes

merged image
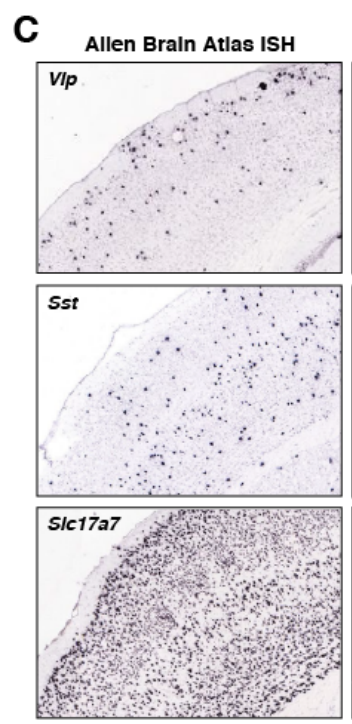

Recovered from composites

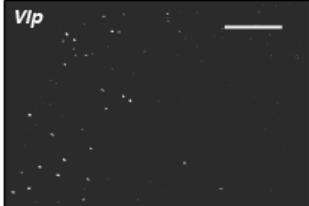

Sst

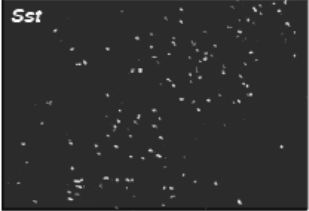

S1c17e7

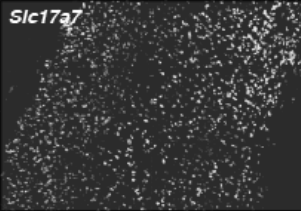

D
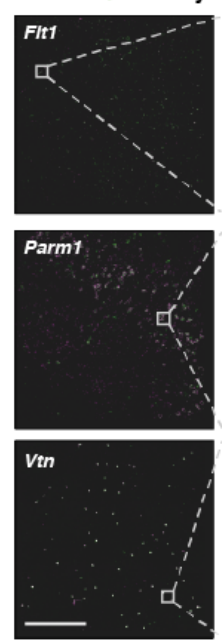
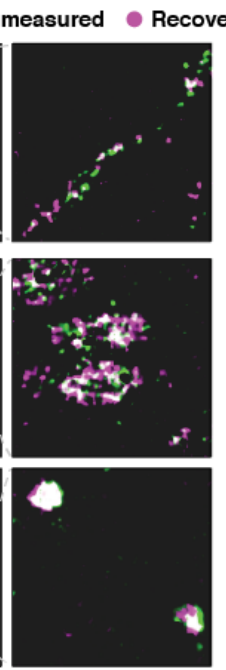

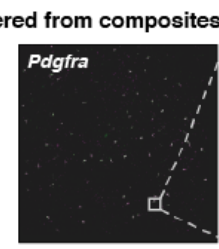

OOverlap
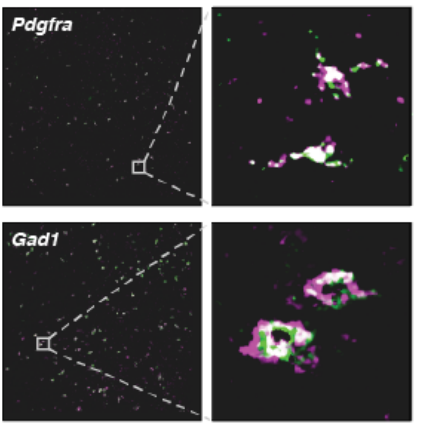

Figure 2. CISI recovers accurate spatial expression patterns from composite experiments 
(a,b) Quantitative accuracy of the composite imaging. (a) A composite image of 12 genes (“Composition 2") compared to a computational merge of 12 images for each individual gene ("Merged image"). 3 of the 12 individual gene images are shown for reference. Left: entire FOV; Right: zoomed in segment, as indicated. Scale bar: 500um. (b) The integrated signal intensity in each segmented cell (individual dots) in the composite image ( $x$ axis) and the merged image $(y$ axis). Pearson's $r$ is noted in upper left corner. (c,d) Autoencodeer based decompression successfully recovers accurate spatial patterns of individual genes. (c) Agreement with canonical expression patterns. Spatial RNA expression for Vip (top), Sst (middle), Slc17a7 (bottom) by ISH (left; Allen Brain Atlas) and in the recovered images by the segmentation free algorithm (right). Scale bar: 500um. (d) Agreement with individual gene measurements on the same section. RNA images recovered by decompression with the segmentation free algorithm (magenta) and directly measured (green) in the same tissue section. White: images overlap exactly. Scale bar 500um. See also Supplementary Fig. 4 for additional genes. 
bioRxiv preprint doi: $\mathrm{https}$ //doi.org/10.1101/743039; this version posted August 24 2019. The copyright holder for this preprint (which was not certified by peer review) is the author/funder, who has granted bioRxiv a license to display the preprint in perpetuity. It is made available under aCC-BY-NC 4.0 International license.

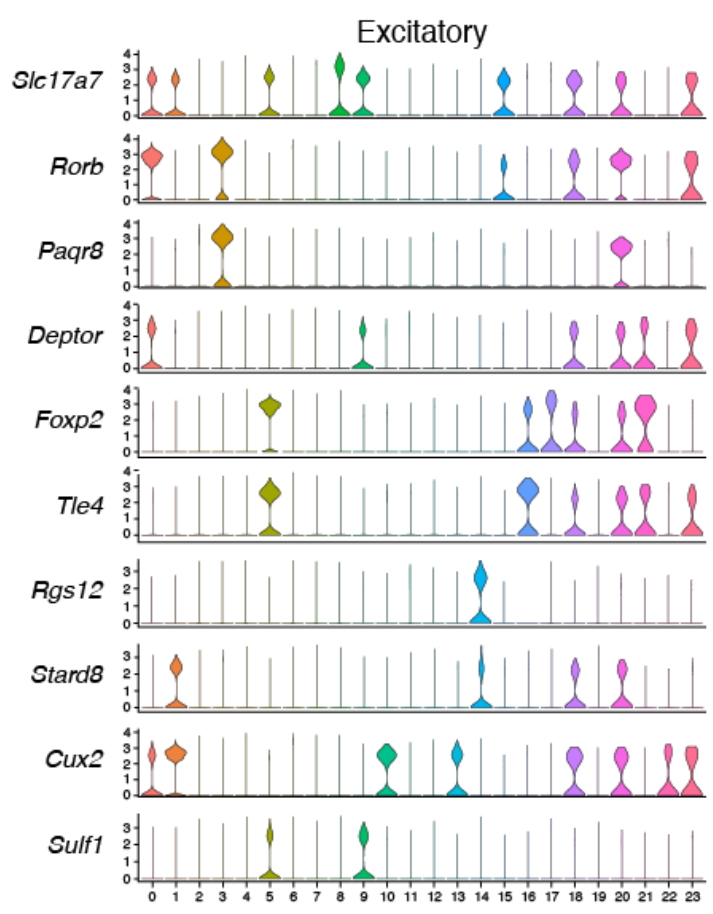

Astrocytes
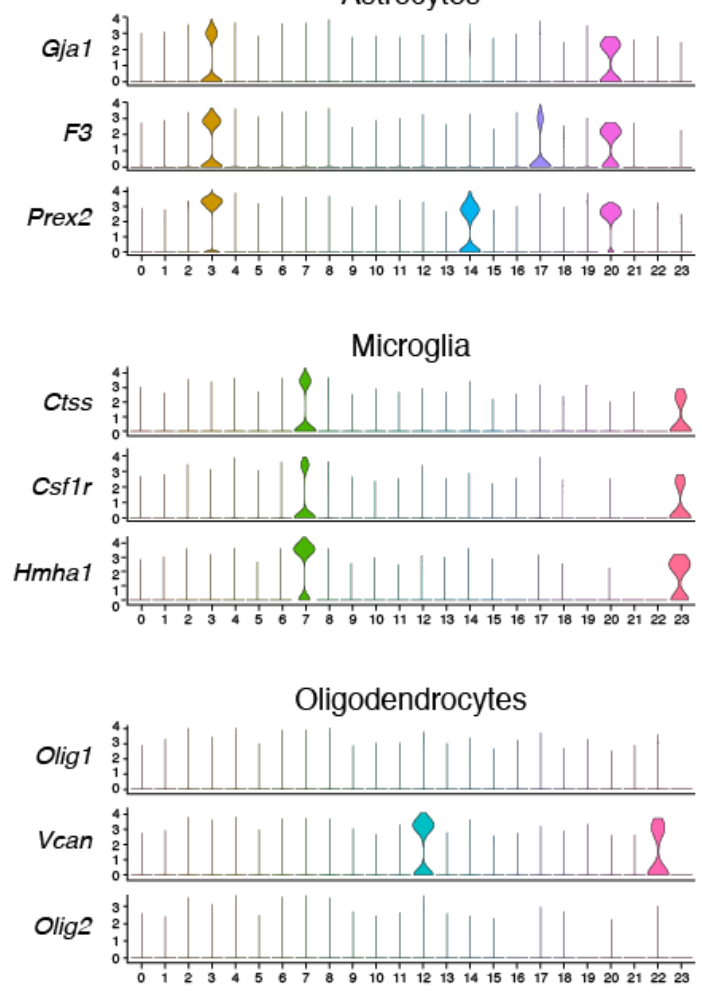

Oligodendrocyte precursors

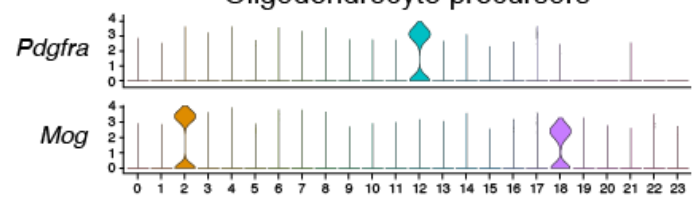

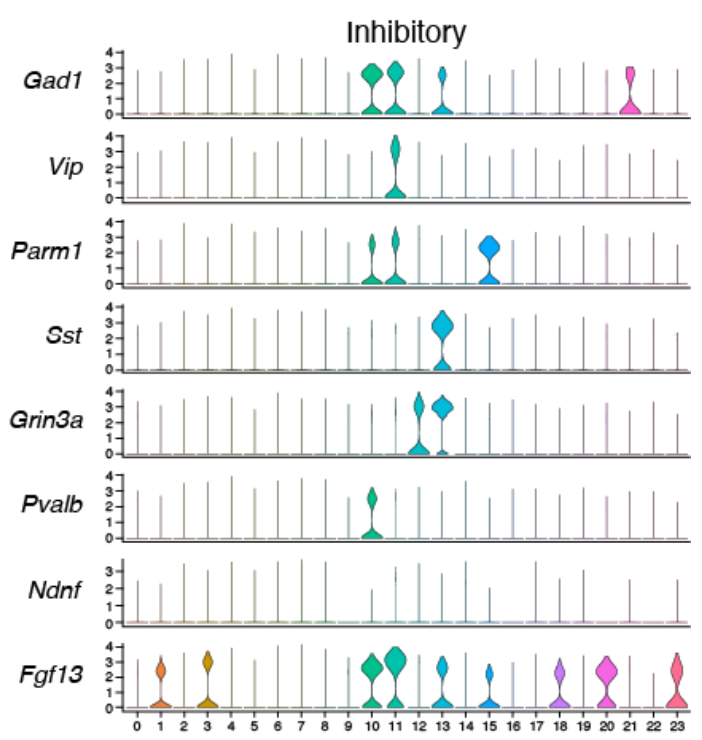

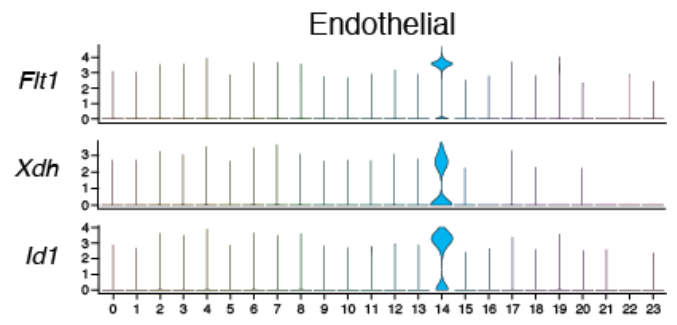

SMC
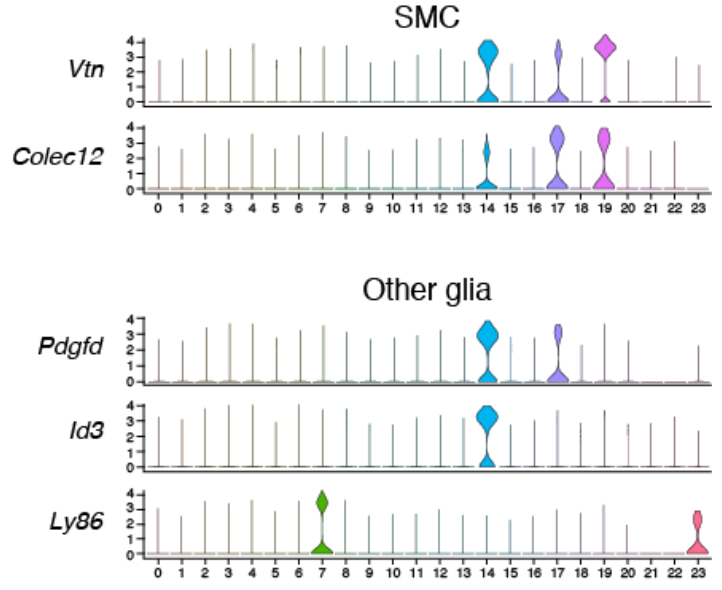

Supp. Fig. 1 


\section{Supplementary Figure 1. Marker gene expression in snRNA-seq clusters}

For each of 37 genes, shown is the distribution of expression (individual violin plots; y-axis) in each of 23 snRNA-Seq clusters ( $x$ axis). Marker genes for similar cell types are grouped together with the cell type labeled on top.

\section{Supp. Fig. 2}
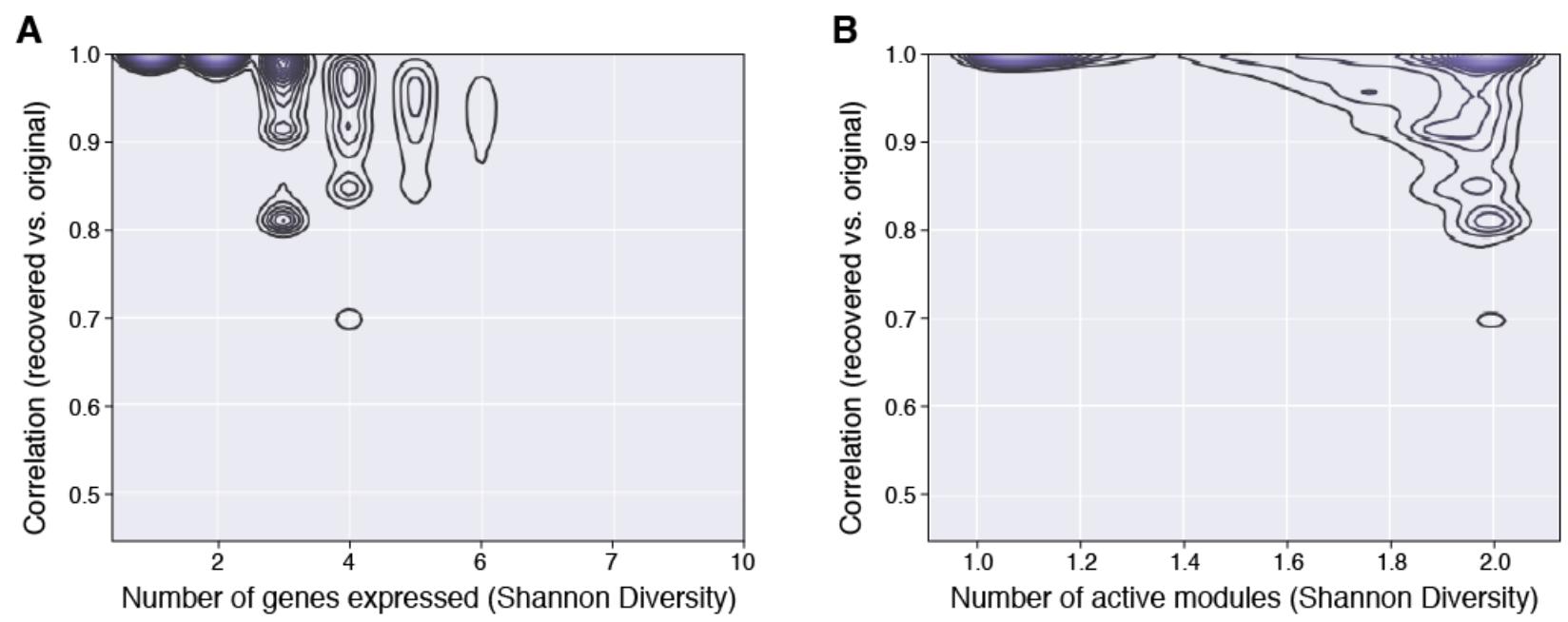

\section{Supplementary Figure 2. Analysis of modular factorization based on gene and module diversity}

Pearson correlation (y-axis) between the original expression levels of 37 genes in each cell and those approximated in those cells by Sparse Module Activity Factorization (SMAF). Contour plots depict the density of cells at each level of correlation with either a given number of genes expressed (a; x-axis) or a given number of gene modules by SMAF decomposition (b; x-axis). 


\section{Supp. Fig. 3}

A

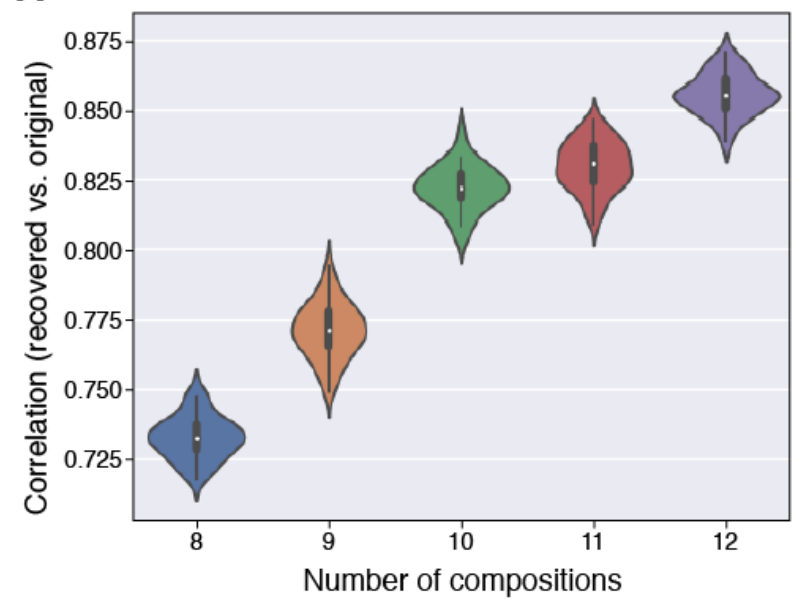

B

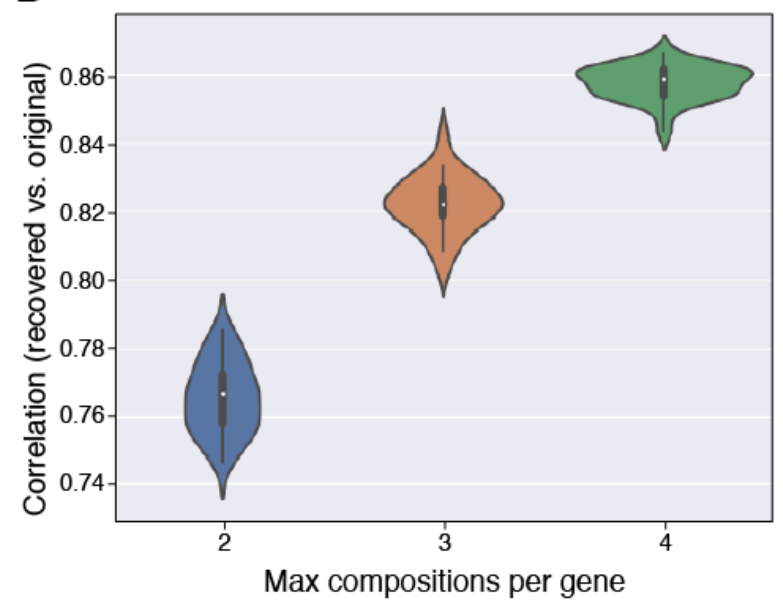

\section{Supplementary Figure 3. Evaluation of performance of simulated compositions}

Distribution of Pearson correlation between the original and recovered expression levels of 37 genes in each cell (y axis) across simulation trials for different numbers of composite measurements (a), or for different measurement densities, set by the maximum number of measurements in which each gene was included (b). In (a) the maximum compositions per gene is 3, and in (b) the number of compositions is 10. Mini boxplots depict median (dots), inner quartiles (box), and 1.5x quartile range (whiskers). 


\section{Supp. Fig. 4}
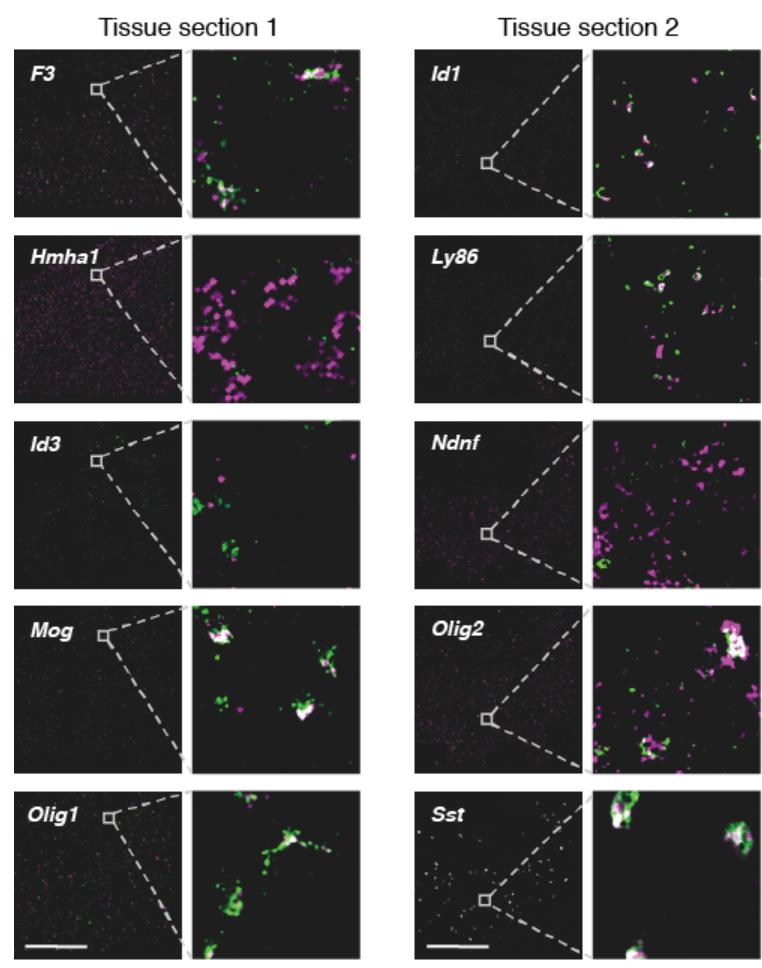

Tissue section 5

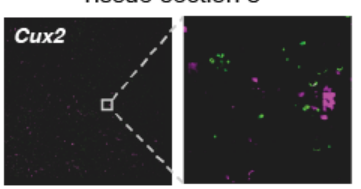

Tissue section 6
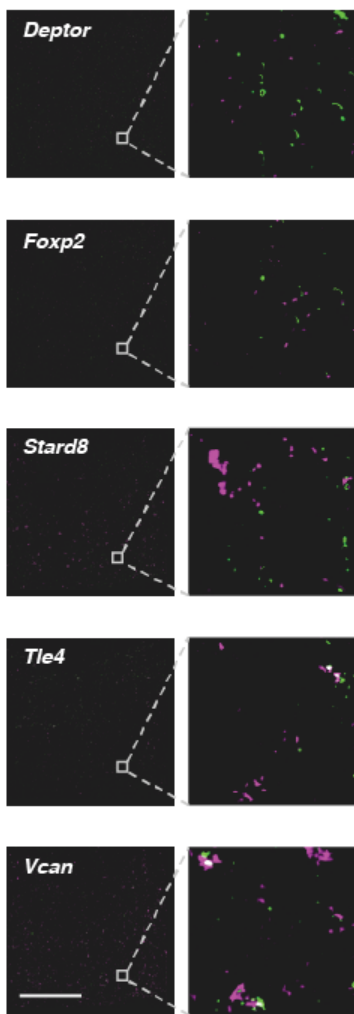
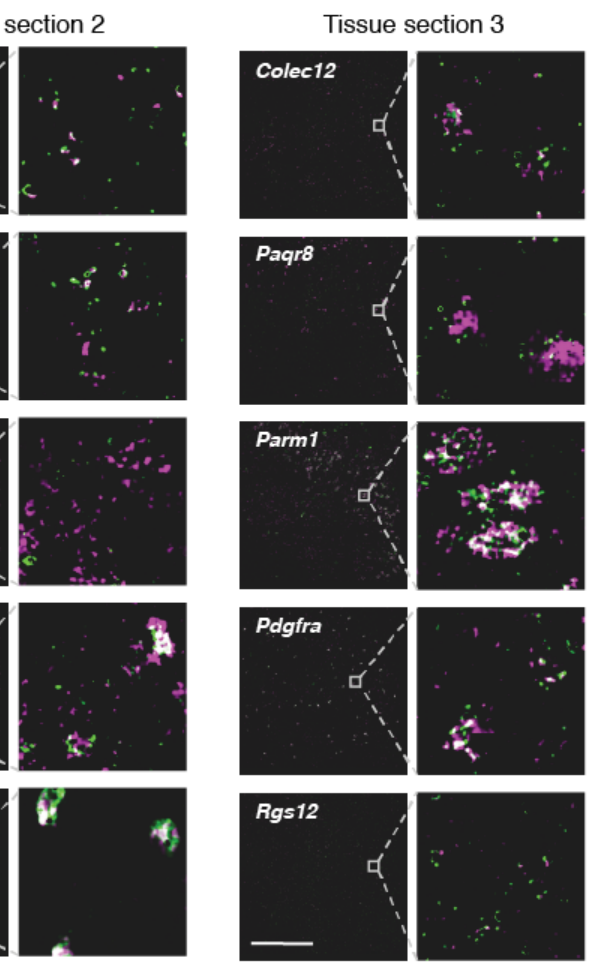

\section{Tissue section 7}
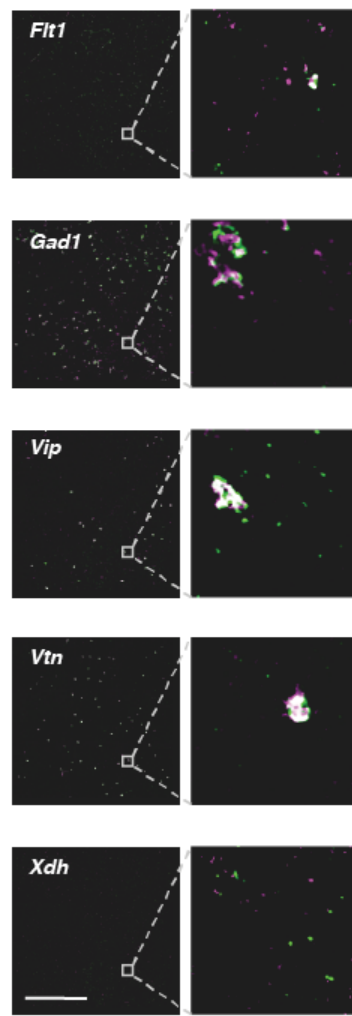
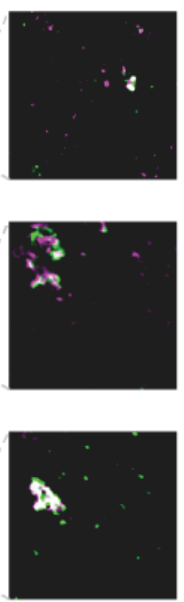

Directly measured

- Recovered from composites

D Overlap
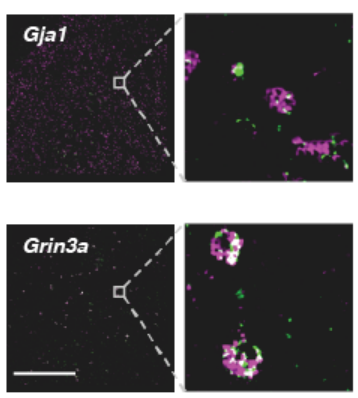
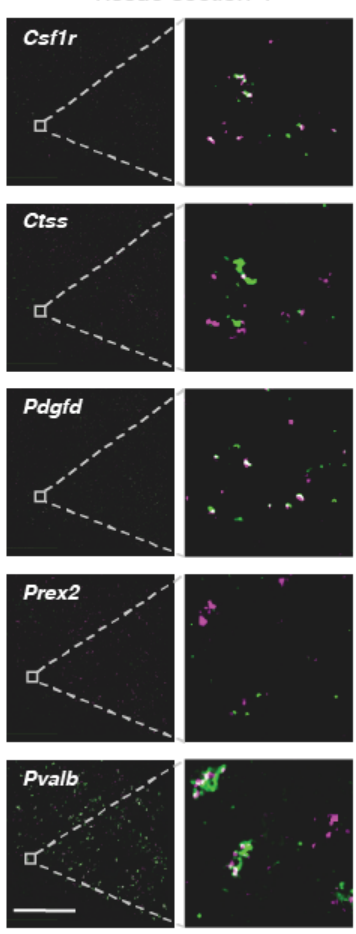

\section{Tissue section 8}
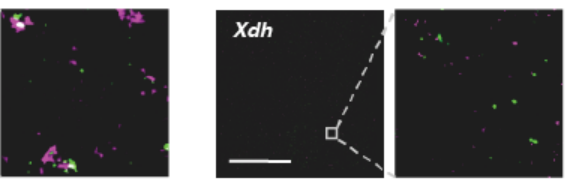


\section{Supplementary Figure 4. Autoencodeer based decompression successfully recovers accurate} spatial patterns of individual genes compared to direct measurement on the same section.

RNA images recovered by decompression with the segmentation free algorithm (magenta) and directly measured (green) in the same tissue section. White: images overlap exactly. Genes are grouped based on the section in which their direct measurements were made. Insets for all genes in a section show the same region, or an adjacent region if no cells for a given gene were present. Scale bar: 500um. 


\section{Supp. Fig. 5}
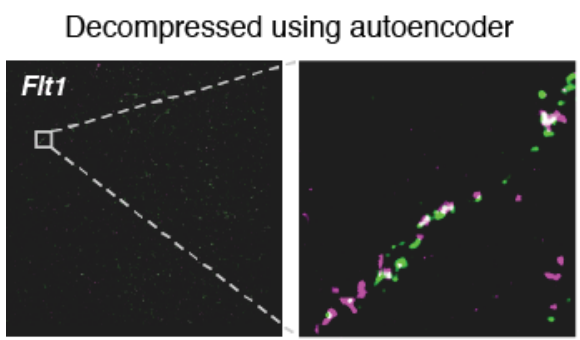

Decompressed using segmentation
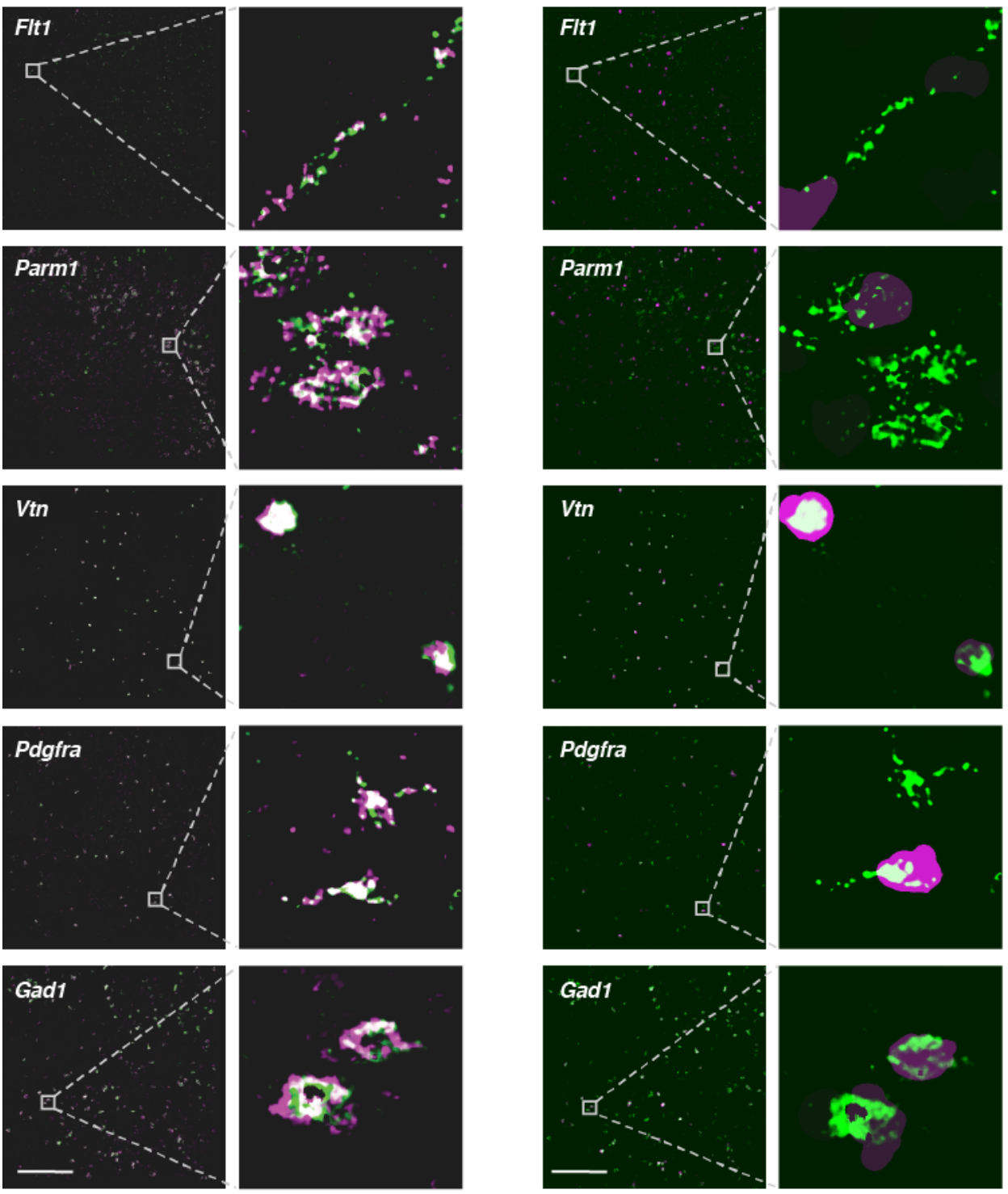

Directly measured Recovered from composites
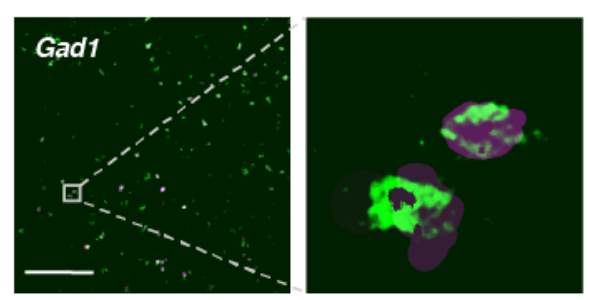

DOverlap

Supplementary Figure 5. Comparison of autoencoding and segmentation-based

\section{decompression}

Individual gene images recovered (magenta) using the autoencoding algorithm (left) or the segmentation based algorithm (right) are overlaid with direct measurement (green) of the genes in the same tissue sections (white: direct overlap). For segmentation-based decompression, the 
bioRxiv preprint doi: https://doi.org/10.1101/743039; this version posted August 24, 2019. The copyright holder for this preprint (which was not certified by peer review) is the author/funder, who has granted bioRxiv a license to display the preprint in perpetuity. It is made available under aCC-BY-NC 4.0 International license.

decompressed signal for each gene is projected uniformly over each segmentation mask. Scale bar:

500um. 
bioRxiv preprint doi: https://doi.org/10.1101/743039; this version posted August 24,2019 . The copyright holder for this preprint (which was not certified by peer review) is the author/funder, who has granted bioRxiv a license to display the preprint in perpetuity. It is made available under aCC-BY-NC 4.0 International license.

\section{A Before adjustment}

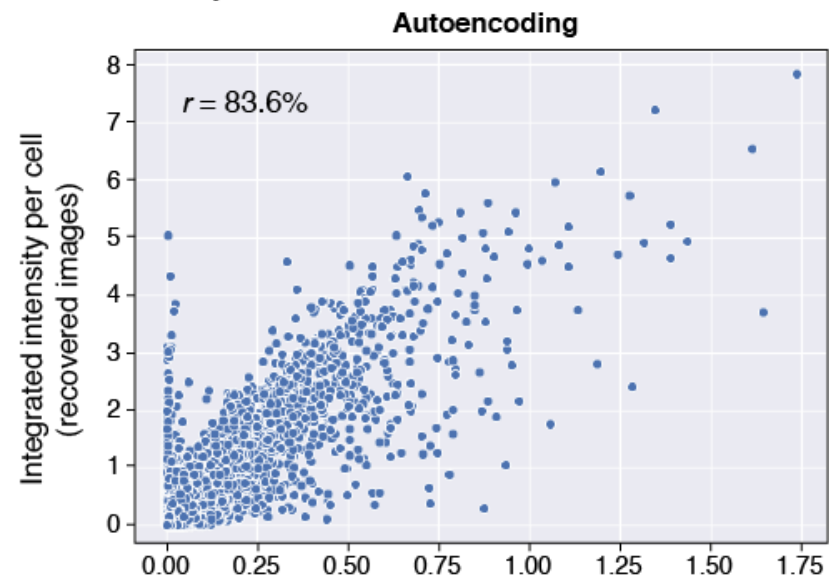

B After adjustment
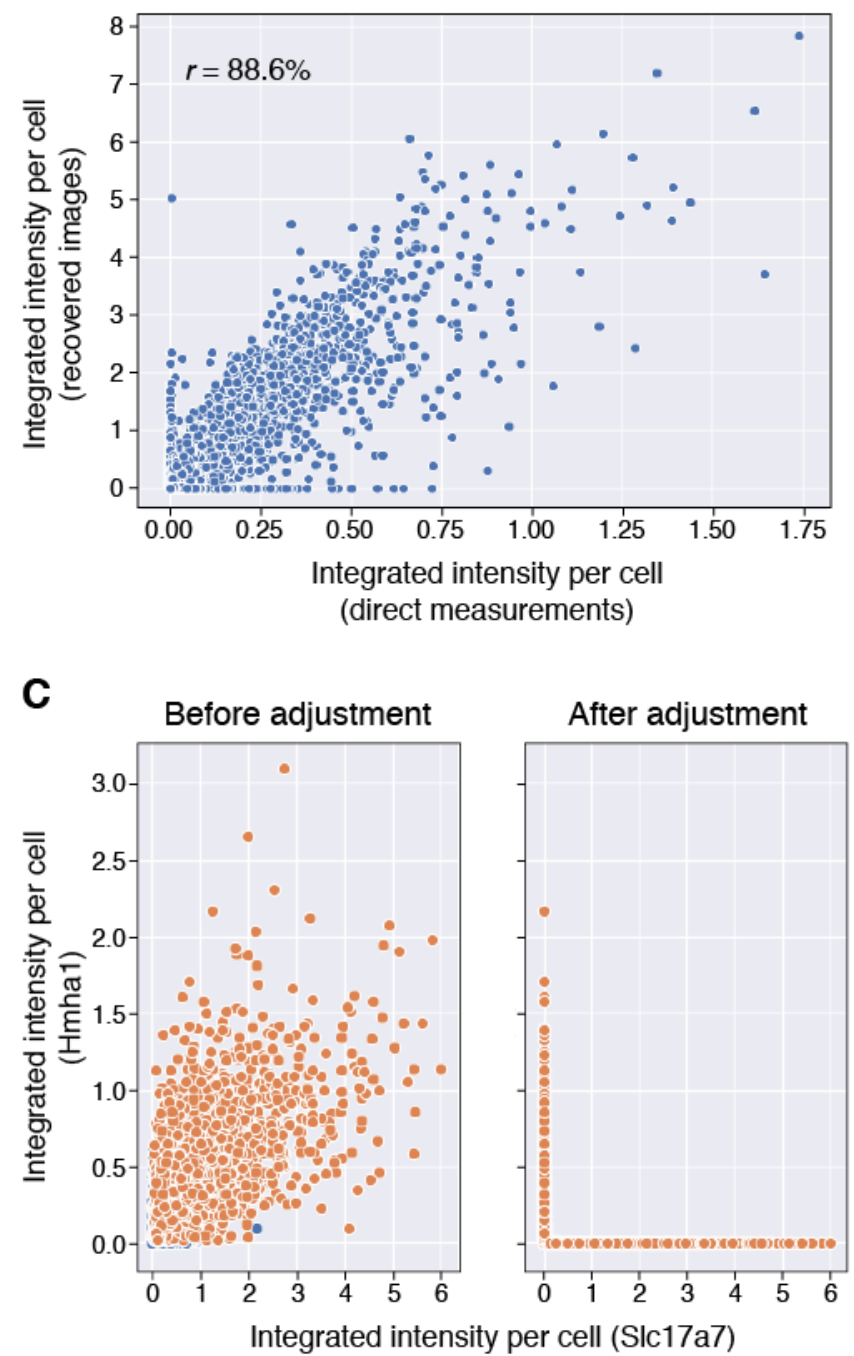
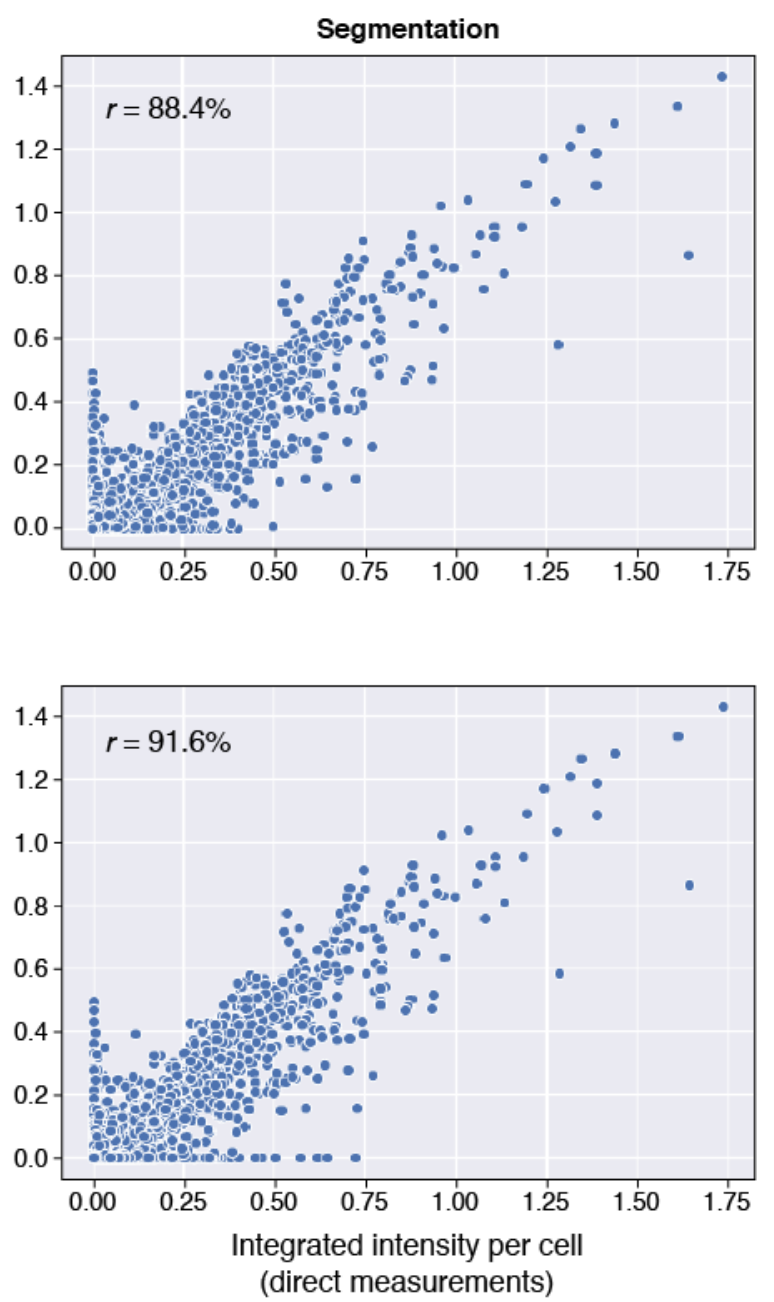

\section{Supp. Fig. 6}




\section{Supplementary Figure 6. Evaluation of recovered signals before and after co-measurement}

\section{adjustment}

(a,b) Adjustment improves recovered signals. Integrated signal intensity for each gene in each cell

(individual dots) from direct measurements ( $\mathrm{x}$ axis) and from estimates recovered by the autoencoder decompressed images (y axis) either before (a) and after (b) co-measurement correction. (c) Example correction. Segmented cell intensities before (left) and after (right) correction for two co-measured genes (Hmhal and Slc17a7) that were not correlated in snRNASeq.

\section{Supp. Fig. 7}
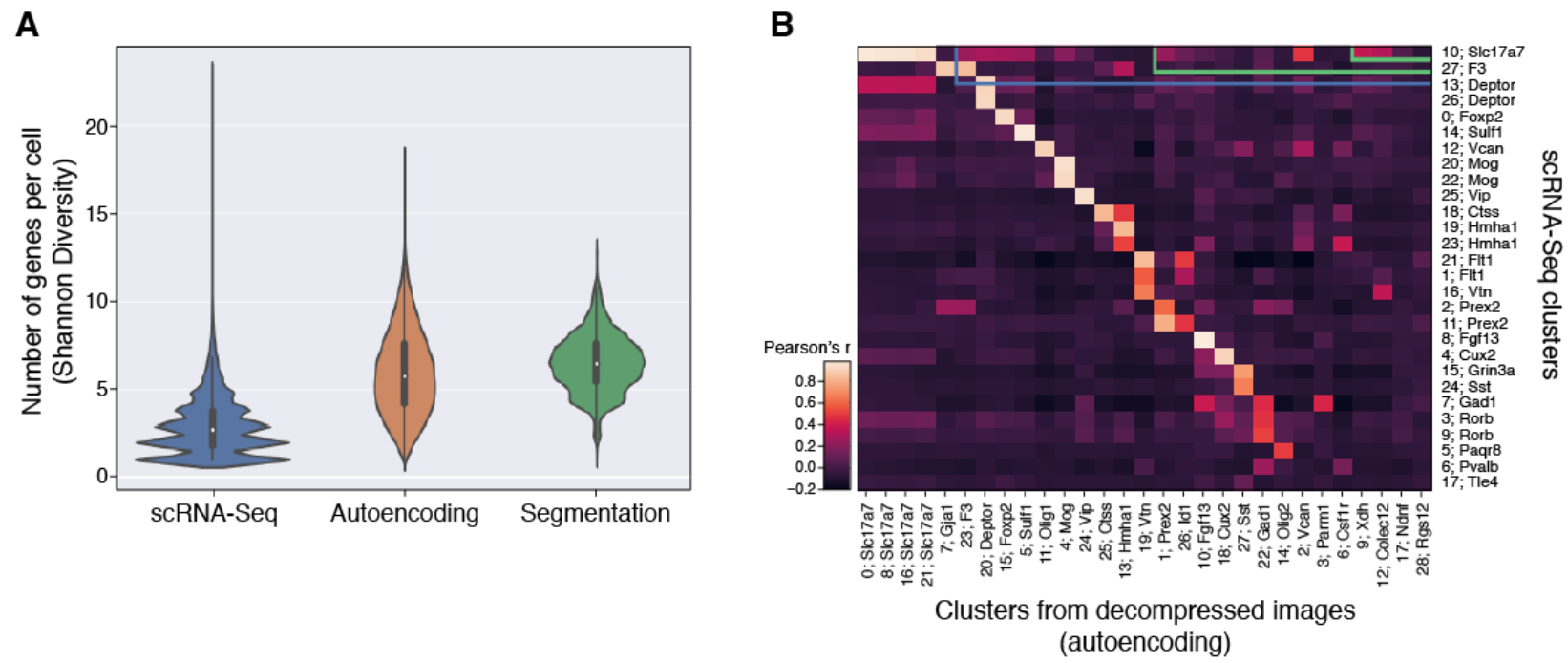

\section{Supplementary Figure 7. Evaluation based on genes per cell and cell clusters}

(a) Distribution of expression diversity (effective number of genes per cell; $y$ axis) in snRNA-Seq, or based on recovered expression levels using autoencoding or segmentation-based decompression ( $x$ axis). Mini boxplots depict median (dots), inner quartiles (box), and 1.5x quartile range (whiskers). (b) Correspondence (Pearson's correlation of mean gene expression; color bar) between cell clusters from snRNA-Seq (rows) and those found from post hoc segmentation of 
bioRxiv preprint doi: https://doi.org/10.1101/743039; this version posted August 24,2019 . The copyright holder for this preprint (which was not certified by peer review) is the author/funder, who has granted bioRxiv a license to display the preprint in perpetuity. It is made available under aCC-BY-NC 4.0 International license.

images recovered using the autoencoding algorithm (columns). One marker gene for each cluster is indicated. 


\section{Methods}

Mice

All mouse work was done with an adult C57B/L6 mouse according to IACUC procedures specified on protocol 0211-06-18.

\section{Analysis of single-nucleus RNA-Seq data}

We selected 37 cell type/layer-specific markers by analyzing snRNA-seq data sets released by BICCN (U19 Huang generated by Regev lab; http://data.nemoarchive.org/biccn/lab/regev/transcriptome/sncell/) for mouse primary motor cortex (M1 or MOp) and generated using the 10x single-cell 3' protocol (V2). To align the reads, a custom reference was created by $10 \mathrm{X}$ Cell Ranger (v.2.0.1, 10X Genomics) using mouse genome and pre-mRNA annotation (Mus_musculus.GRCm38, release 84) according to the instructions provided on the 10X Genomics website (https://support.10xgenomics.com/single-cell-geneexpression/software/release-notes/build\#mm10_1.2.0). The default parameters were used to align reads, perform UMI counting, filter high quality nuclei and generate gene by nucleus count matrices. In total, 30,000 nuclei passed QC metrics including (i) the number of unique genes detected in each cell $(>200)$ and (ii) the percentage of reads that map to the mitochondrial genome $(<10 \%)$, and featured in the further downstream analyses using the Seurat package (version 2.2.1).

\section{Compressed sensing simulations}

We use compressed sensing to recover sparse signals from composite measurements. In the basic formulation, we seek to recover sparse gene module activities, $W \in \mathbb{R}^{d x n}$, and estimate unobserved gene abundances, $U W=\hat{X} \in \mathbb{R}^{g \times n}$ given observations $A U W=Y \in \mathbb{R}^{m \times n}$, a gene 
module dictionary $U \in \mathbb{R}^{g x d}$, and measurement compositions $A \in \mathbb{R}^{m \times g}$, where there are $m$ composite measurements of $g$ genes in each of $n$ cells, and the dictionary consists of $d$ gene modules.

Using the snRNA-Seq data above, and the 37 selected genes, we evaluated different composite designs by simulating composite measurements and recovering individual expression levels by sparse optimization (as previously described(Cleary et al., 2017); see also https://github.com/cleary-lab/CISI). Briefly, we first randomly selected training, validation and testing subsets, using $60 \%, 20 \%$, and $20 \%$ of all cells for each respective group. In the training set, we calculated a dictionary, $U \in \mathbb{R}^{g x d}$, with $d=80$ modules of $g=37$ genes (and default SMAF parameters found on https://github.com/cleary-lab/CISI). A given simulation trial with $m$ measurements consists of (i) randomly assigning genes to compositions, $A$; (ii) simulating noisy composite measurements in validation data, $Y=A(X+\epsilon)$ (with a signal-to-noise ratio of 5); (iii) decoding sparse module activity levels, $\min _{W}\|W\|_{1} ;$ s.t. $\frac{\|Y-A U W\|}{\|Y\|}<\lambda$; (iv) estimating individual expression levels, $\hat{X}=U W$; and (v) calculating the correlation between the original and estimated levels, corr $=$ Pearson $(X, \hat{X})$. When evaluating different measurement designs in step (i), we varied the total number of measurements (from 8 to 12), and the maximum number of measurements in which each gene appeared (either 2, 3, or 4). Each gene was then randomly assigned to a randomly chosen number of measurements (up to the maximum). Final assignments resulting in either two or more genes being perfectly co-assigned or in large measurement imbalance (any gene appearing more than 4 times more frequently than any other gene) were excluded. We then iterated steps (i)-(v) 2,000 times, selected the 50 composition matrices resulting in the top correlations, and evaluated (steps (ii)-(v)) in testing data. The correlations in testing data 
were then used to compare different numbers of measurements, and maximum assignments per gene (fig. S3).

\section{Selection of the final library}

Based on these comparisons (fig. S3), and considering the number of probes that would need to be synthesized in each scenario, we selected the composition with the highest performance in testing data among those with 10 measurements and a maximum of 3 assignments per gene. Each of the 10 compositions was assigned to one of three colors, to be imaged during 3 1/3 rounds (table S2).

\section{Probe design and validation}

For each target mRNA, HCRv3.0 DNA probe sets of $\sim 20$ probe pairs each were ordered from Molecular Technologies. All HCR v3.0 reagents are now only available from Molecular Instruments, Inc. (molecularinstruments.com). Target binding site sequences can be found in table

\section{S4.}

\section{Tissue preparation and brain extraction}

An adult C57B/L6 mouse was perfused with ice-cold PBS (10010023, ThermoFisher Scientific) prior to dissection of the brain. The brain was then extracted and flash frozen in liquid nitrogen. After OCT embedding, the brain was sectioned directly into an APTES coated 24-well glass bottom plate (82050-898, VWR). For coating, plates were coated with a 1:50 solution of APTES (440140, Sigma) in 100\% Ethanol (V1016, DeconLabs) for 5 minutes followed by 3x washes with 100\% ethanol before drying. Tissues were fixed in 10\% Formalin (100503-120, VWR) for 15 
minutes and washed with PBS before overnight permeabilization with $70 \%$ ethanol. Tissues were re-hydrated with PBS prior to hybridization.

\section{In situ hybridization}

In situ HCR version 3.0 with split-initiator probe sets was performed using the protocol detailed in(Choi et al., 2018) with some slight adaptations. Probe sets for each individual target mRNA were diluted to the concentration specified in the protocol and organized into composite channels. A composite channel is comprised of a mix of probe sets for approximately 10 different target mRNAs, each with the same initiator. In total, 10 composite channels were created. Three composite channels can be hybridized per round of imaging. Thus, for the first round of hybridization, probe sets for three composite channels with distinct initiator sequences were added at once to each tissue.

Probes were hybridized for approximately 8 hours in hybridization buffer and then tissues were washed 4 times with $30 \%$ wash buffer for 15 minutes each and 3 times with 5 X SSCT for 5 minutes each (buffer compositions available from Molecular Instruments). Snap-cooled hairpins were added at a 1:500 diluted concentration and amplification was allowed to proceed for 8 hours. Excess hairpins were then washed off with 5X SSCT (15557044, ThermoFisher Scientific), with $0.2 \%$ Tween-20 for three washes of 15 minutes each. Tissues were stained with DAPI (1:5,000 TCA2412-5MG, VWR) immediately prior to imaging. After imaging, probes were stripped from tissues using $80 \%$ formamide at $37^{\circ} \mathrm{C}$ for 30 minutes. This entire process (hybridization, amplification, imaging, stripping) was repeated for up to five rounds of imaging (see table S5 for composites and individual targets imaged in each round). All DNA HCR amplifiers (hairpins), 
hybridization buffers, wash buffers, and amplification buffers were ordered from Molecular Technologies. All HCR v3.0 reagents are now only available from Molecular Instruments, Inc. (molecularinstruments.com).

\section{Imaging}

Imaging was performed on a spinning disk confocal microscope (Yokogawa W1 on Nikon Eclipse Ti) equipped with a Nikon CFI APO LWD 40x/1.15 water immersion objective operating NISelements AR software with Andor Zyla 4.2 sCMOS detector. DAPI fluorophores were excited with a 405nm laser, Alexa 488 HCR amplifiers were excited with a 488nm laser with 525/36 emission filter (Semrock, 77074803), Alexa 546 HCR amplifiers were excited with a 561nm laser with a 582/15 emission filter (Semorck, FF01-582/15-25), and Alexa 647 HCR amplifiers were excited with a 640nm laser with a 705/72 emission filter (Semorck, 77074329).

\section{Image processing}

Before downstream analysis, we ran a series of image processing steps to normalize, stitch, align, and segment the images in each color, field of view, round, and tissue. We first took a maximum projection across the z-axis, and then used the DAPI channel to stitch the fields of view within each round of imaging (using ImageJ software(Abràmoff et al., 2004)). We applied the stitching coordinates from the DAPI channel to each of the other channels. We then smoothed the image for each channel using a median filter (with a width of 8 pixels). (If spot-level resolution is needed, this step may not be advised. Since we do not need this resolution, we use this step to make autoencoder reconstruction an easier task.) From each smoothed image, we aligned and subtracted background signal, obtained by imaging after stripping the final round of fluorescent probes. We 
then adjusted brightness and contrast by rescaling according to upper and lower thresholds determined using auto-adjust in ImageJ. The same rescaling parameters for each channel (determined from the maximum upper threshold and minimum lower threshold) were applied to all tissues and rounds. After rescaling, we applied a flat field correction to each field of view, by normalizing (dividing) each pixel by the median smoothed pixel intensity across all images (with smoothing by a Gaussian filter with a width $1 / 8$ of the image dimension). Each round of the flat field-corrected images in a given tissue was then aligned using ImageJ. These images were used in the remainder of downstream analysis.

For segmentation, we used CellProfiler(McQuin et al., 2018), and calculated one image mask per nucleus in each tissue using DAPI in the first round. Each mask was then expanded by up to 10 pixels (without overlapping a neighboring cell). Comparisons and decompression with segmented cells were done using the integrated image intensity in each expanded nucleus mask.

\section{Decompression of composite signals}

We developed two methods to decompress composite signal intensities into signals for individual genes.

The first method, which we used primarily as a point of reference for validation statistics, is based on cell segmentation. Given the intensities of each composite measurement in each segmented cell, $Y \in \mathbb{R}^{m x n}$, we solved a sparse optimization problem to decode sparse module activity levels, $\min _{W \in \mathbb{R}^{d x n}}\|W\|_{1} ; \quad$ s.t. $\frac{\|Y-A U W\|}{\|Y\|}<\lambda$, before estimating individual expression levels, $\hat{X}=$ $U W$, with the same method as in our simulations (above). 
The second method we developed decompresses entire images using a convolutional autoencoder. In this approach, for a given set of 10-channel composite images, we first train a model to identify a reduced (encoded) representation of each image, which can then be decoded to recapitulate the original. During this training, we optimize the following loss function:

$$
\|\log (Y+\varepsilon)-\log (\hat{Y}+\varepsilon)\|_{1}+\lambda_{\text {pixel }} \mathcal{L}_{\text {pixel }}+\lambda_{T V} \mathcal{L}_{T V}
$$

where $Y$ is the original image, $\hat{Y}$ is the decoded image, $\mathcal{L}_{\text {pixel }}$ is a loss on pixel density, $\mathcal{L}_{T V}$ is the total variation of $\hat{Y}, \lambda_{\text {pixel }}$ and $\lambda_{T V}$ are hyperparameters, and $\varepsilon$ is a small constant. $\mathcal{L}_{\text {pixel }}$ is calculated as the Poisson log-likelihood of the pixel density, which is computed as the Shannon Diversity across pixels, divided by the number of pixels, with prior density set by a parameter $\delta_{\text {pixels }}$. Convolutions in each layer of the network are computed across filters (or kernels), but not across the 10 composite channels. Hence, each of the 10 channels remains separated from the other channels throughout each layer of the network. However, only one set of convolutional weights is learned; these are shared across all channels. The number of parameters in the model is, thus, relatively small, and the autoencoder trained quickly on our data. As discussed below, hyperparameters, including the number of encoding and decoding layers, the number and size of filters, and pooling sizes are chosen by hyperparameter tuning on a small set of validation images.

Using the trained autoencoder, we decompress composite images as follows. First, we encode each 10-channel image to a reduced representation, $\tilde{Y} \in \mathbb{R}^{10 x \widetilde{w} \times \widetilde{h} \times f}$, where $\widetilde{w}$ and $\tilde{h}$ are the reduced width and height (after pooling at each encoding layer), and $f$ is the number of convolutional filters. We then solve for sparse module activities, $\widetilde{W} \in \mathbb{R}^{d x \widetilde{w} \times \widetilde{h} x f}$, where $d$ is the number of modules in the dictionary (here, 80), and then estimate the encoded representation of each 
individual (unobserved) gene, $\tilde{X} \in \mathbb{R}^{37 x \widetilde{w} x \widetilde{h} x f}$. These representations are then run through the pre-trained decoder to produce an image for each gene, $\hat{X} \in \mathbb{R}^{37 \times w \times h}$.

Our loss function has components at both the encoding and decoding layers:

$$
\mathcal{L}=\|\tilde{Y}-A U \widetilde{W}\|_{2}^{2}+\|\log (Y+\varepsilon)-\log (A \hat{X}+\varepsilon)\|_{1}+\lambda_{\text {pixel }} \mathcal{L}_{\text {pixel }}+\lambda_{T V} \mathcal{L}_{T V}+\lambda_{W} \mathcal{L}_{W},
$$

where $\mathcal{L}_{W}$ is a loss on the density of $\widetilde{W}$, calculated as the Poisson log-likelihood of the Shannon diversity, with prior density set by parameter $\delta_{W}$. We implemented our model in tensorflow, using the Adam optimizer.

Hyperparameters and model architectures were chosen by hyperparameter tuning on a small set of validation images. The validation images consist of 4 of 36 patches from each of 3 images (i.e., from 3 tissue sections, each with 36 patches), for a total of 12 patches (equivalent in size to $1 / 3$ of one image). Each patch includes signal from the 10 composite measurements, along with up to 5 directly measured genes. In each validation trial, we select hyperparameters, train the autoencoder on the composite data, decompress all genes, and then calculate the trial score as the correlation between the subset of directly measured and recovered genes (this is done in post hoc segmented cells, when using the autoencoder). We selected the hyperparameters from the best performing trial, and used these to run our analysis on the full dataset. More details can be found at https://github.com/cleary-lab/CISI.

We applied a heuristic correction to co-measured genes. We first identified 104 pairs of comeasured genes (i.e., that co-occur in more than one composition) that were not correlated $(<10 \%)$ in snRNA-Seq. For each pair, we set the expression of one of the two genes to zero whenever they 
were co-expressed in recovered images. To select which gene to adjust, we calculate the correlation between the 10 composite measurements in a cell, and the pattern of measurements for each of the two genes (e.g., the binary vector indicating which measurements included the gene), and then adjust to zero the gene with the lower correlation.

\section{Plotting decompressed images}

The decompressed results for each gene vary in their relative signal intensities (as do direct measurements for each gene). When plotting merged validation images (as in fig. $2 \mathrm{~d}$ and fig. S4), we normalize the signal for each gene to automatically adjust contrast and brightness. The specific parameters of this normalization can be found in the code demo of our online repository (https://github.com/cleary-lab/CISI/blob/master/getting_started/plot_decompressed_images.py).

The signal plotted for direct measurements have been pre-processed according to the methods described above.

\section{Comparison of CISI and combinatorial barcoding}

We can approximate the number of imaging rounds in CISI, $\mathrm{r}_{\mathrm{CISI}}$, relative to that in combinatorial

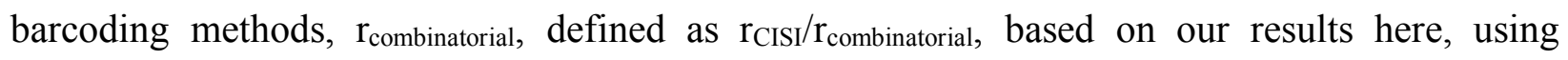
simulation to extrapolate to larger scales, and by comparing with existing combinatorial methods. Here, we used 3 and a 1/3 rounds to measure 37 genes; the same could be achieved using 3 -color combinatorial barcoding without error correction. More commonly, 4 or 5 rounds would be used to measure 37 genes and allow for error correction. At larger scales, simulations in our earlier work (Cleary et al., 2017) suggest that $\sim 100$ composite measurements would suffice to approximate the expression of 10,000 genes. This could be done in 33 and a 1/3 rounds of CISI. To date, the only 
bioRxiv preprint doi: https://doi.org/10.1101/743039; this version posted August 24, 2019. The copyright holder for this preprint (which was not

certified by peer review) is the author/funder, who has granted bioRxiv a license to display the preprint in perpetuity. It is made available under aCC-BY-NC 4.0 International license.

combinatorial method to scale to this level did so with 80 rounds of imaging(Eng et al., 2019). We therefore very roughly approximate that the required rounds of imaging with either approach will be comparable, and that $\mathrm{r}_{\mathrm{CISI}} / \mathrm{r}_{\text {combinatorial }}$ will be in the range $1 / 3$ to 3 , allowing for improvements in combinatorial methods and the possibility of needing more rounds than anticipated with CISI. 